\title{
Ageing of a Microscopic Sliding Gold Contact at Low Temperatures
}

\author{
Nitya Nand Gosvami, ${ }^{1}$ Michael Feldmann, ${ }^{2}$ Joël Peguiron, ${ }^{3}$ Michael Moseler, ${ }^{3}$ \\ André Schirmeisen, ${ }^{4}$ and Roland Bennewitz ${ }^{1}$ \\ ${ }^{1}$ INM - Leibniz Institut für Neue Materialien, Campus D2 2, 66123, Saarbrücken, Germany \\ ${ }^{2}$ Physikalisches Institut and Center for Nanotechnology (CeNTech), Westfälische Wilhelms-Universität Münster, \\ Wilhelm-Klemm-Strasse 10, 48149 Münster, Germany \\ ${ }^{3}$ Fraunhofer-Institut für Werkstoffmechanik IWM, Wöhlerstrasse 11, 79108 Freiburg, Germany \\ ${ }^{4}$ Institute of Applied Physics, Justus-Liebig Universität Giessen, Heinrich-Buff-Ring 16, 35392 Giessen
}

(Received 10 June 2011; published 30 September 2011)

\begin{abstract}
Nanometer-scale friction measurements on a $\mathrm{Au}(111)$ surface have been performed at temperatures between 30 and $300 \mathrm{~K}$ by means of atomic force microscopy. Stable stick slip with atomic periodicity is observed at all temperatures, showing only weak dependence on temperature between 300 and $170 \mathrm{~K}$. Below $170 \mathrm{~K}$, friction increases with time and a distortion of the stick-slip characteristic is observed. Low friction and periodic stick slip can be reestablished by pulling the tip out of contact and subsequently restoring the contact. A comparison with molecular dynamics simulations indicates that plastic deformation within a growing gold junction leads to the observed frictional behavior at low temperatures. The regular stick slip with atomic periodicity observed at room temperature is the result of a dynamic equilibrium shape of the contact, as microscopic wear damage is observed to heal in the sliding contact.
\end{abstract}

DOI: 10.1103/PhysRevLett.107.144303

PACS numbers: 46.55.+d, 62.20.Qp, 81.40.Pq

Metals carry significant importance for a large variety of applications involving small sliding contacts ranging from structural components and electronic switches to microelectromechanical systems [1]. Despite such wide application, fundamental tribological properties of metals at the microscopic length scale are not very well understood $[2,3]$. Although macroscopic tribological studies of metals have been carried out for a long time, such studies do not provide understanding of microscopic friction and wear mechanisms due to the complex nature of the contact, which almost always involves multiple asperity interactions, plastic deformation, and wear [2]. The invention of the atomic force microscope (AFM) operating in ultrahigh vacuum (UHV) provides a powerful tool to address tribological properties of metals with a rather well defined single asperity contact in a contaminant-free environment, which is crucial to analyze the fundamental mechanisms of friction and wear [4]. Despite using AFM techniques in highly controlled environments and with idealized single asperity contacts on atomically flat terraces of metal surfaces, it is still difficult to carry out reliable friction experiments due to the high surface energy of metals under clean conditions, which leads to high reactivity of surface atoms resulting in cold welding or junction formation under the tip apex [5-7]. Until now, there have been only a few experimental room-temperature studies of friction on metals at the atomic scale [8-10], which showed that for a small range of loads a stable atomically corrugated friction image of clean metal surfaces can be obtained.

In order to develop further insight into the tribological behavior of metals, temperature is an important parameter to address. Temperature has a strong effect on mechanical strength as well as on the mobility of surface atoms. Surface diffusion of atoms could have a significant effect on their frictional properties, as it plays a key role in contact formation and healing of wear damage [11]. The effect of temperature on nanometer-scale friction has only recently been studied for several nonmetallic systems such as polymers, silicon, and layered solids [12-15]. Temperature was found to have a significant effect on nanoscale friction in general and its velocity dependence in particular $[12,14]$ by thermal activation of the underlying microscopic processes. Materials studied so far provide structural stability for a wide range of temperatures, which is crucial for the formation of a stable contact. Surface diffusion adds complexity to nanometer-scale experiments on metals as we report in this Letter.

We explore the friction behavior on atomically flat $\mathrm{Au}(111)$ surfaces at various temperatures and tip scanning velocities, under a constant applied load. Stick-slip events with atomic periodicity are observed at all temperatures from 30 to $300 \mathrm{~K}$ which allows for a view on fundamental mechanisms of friction. Surprisingly, we find only weak influence of temperature on friction down to $170 \mathrm{~K}$. At temperatures lower than $170 \mathrm{~K}$, a regime of increase in friction with time is observed, which we explain with the growth of a Au neck between sliding tip and sample at low temperatures. This interpretation is supported by atomistic simulations of metallic tips sliding on $\mathrm{Au}(111)$.

The instrument used for all experiments is a UHV-AFM (Omicron Nanotechnology, Germany) which allows sample heating and cooling. Forces are measured as the bending of a microcantilever which is detected using the optical beam deflection method. All measurements were 
conducted at a pressure lower than $2 \times 10^{-10}$ mbar. $\mathrm{Au}(111)$ surfaces with $\sim 200 \mathrm{~nm}$ large terraces were prepared by flame annealing of $300 \mathrm{~nm}$ thick gold films on mica under atmospheric conditions. The samples were immediately introduced into the UHV system and were heated above $200^{\circ} \mathrm{C}$ for $1 \mathrm{~h}$ to remove water and adsorbed contaminants. Details on gold film preparation, force sensors, their calibration, and data processing can be found elsewhere [10]. Essentially, the recorded signal of the cantilever twisting provides the actual lateral force, while the friction force is determined by averaging the lateral force over many pairs of forward and backward scans across several unit cells of the crystal structure. In order to avoid wear damage of the $\mathrm{Au}(111)$ surface, we have performed all friction measurements on atomically flat terraces at a constant applied load of $<5.0 \mathrm{nN}$ where stable experiments are possible over extended periods of time [10]. For all results presented in this Letter, the lateral force maps revealed the herringbone reconstruction and exhibited clear atomic-scale modulation.

Figure 1 presents experimental results for friction as a function of scanning velocity for temperatures of 170, 230, and $300 \mathrm{~K}$, under a constant applied normal load of $\sim 3 \mathrm{nN}$. Each data point is the average over scan frames of $6 \times 6 \mathrm{~nm}$. Although there is significant scatter in the data, it can be observed that friction increases with velocity. These velocity dependence experiments are fully reversible for each temperature with an increase or decrease in velocity. Stable nanometer-scale sliding contacts are formed for which stick-slip friction with atomic periodicity at given velocities and temperatures is constant over long scanning times. The dependence of friction on velocity is reasonably well described as logarithmic increase. This is in agreement with previous nanometer-scale experiments on dry friction and can be explained by thermal activation of the friction process $[16,17]$. The dependence of friction

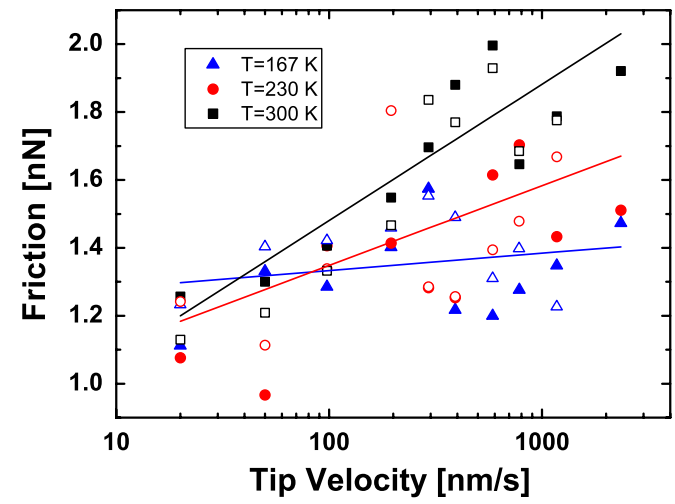

FIG. 1 (color online). Friction as a function of tip velocity recorded on a $\mathrm{Au}(111)$ surface at different sample temperatures. Full symbols indicate data from a series with increasing velocities, open symbols from a subsequently recorded series with decreasing velocities. The lines are fits to the data assuming a logarithmic increase of friction with velocity. on velocity is stronger at higher temperatures and weaker at lower temperatures, again in agreement with the interpretation of thermally activated jump processes. A quantitative comparison between friction results at different temperatures is difficult because each measurement at a newly established temperature involves the formation of a new contact through repeated scanning over several step edges. We can still state that the effect of temperature on friction is not very strong.

The friction behavior on $\mathrm{Au}(111)$ changes dramatically for temperatures lower than $170 \mathrm{~K}$. Regular stick slip with atomic periodicity is initially observed. With time, friction increases and the lateral force curves become more and more distorted. However, low-friction and regular stick slip comparable to the results for higher temperatures can be reestablished by pulling the tip out of contact and bringing it back into contact, just to start another increase in friction with time. Typical experimental results are presented in Fig. 2(a). The increase of friction with time is interrupted by breaking and reforming the contact. The normal force curves in Fig. 2(b) recorded while pulling off the tip show a strong reduction in pull-off force between the high-friction and low-friction regimes. In contrast, we did not observe any change in adhesion with time at temperatures below $170 \mathrm{~K}$ when the tip was not scanned over the surface, suggesting that the increase in friction and adhesion is caused by the sliding of the contact over the $\mathrm{Au}(111)$ surface at low temperatures.

The results indicate a growth of the contact between tip and $\mathrm{Au}(111)$ in the course of sliding at temperatures below $170 \mathrm{~K}$. The growth mechanism is not operational at higher
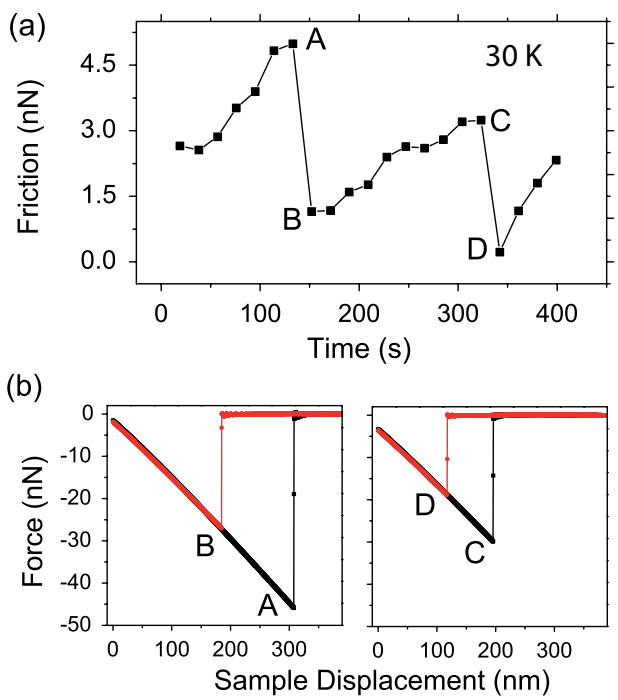

FIG. 2 (color online). (a) Variation of friction as a function of scanning time at lower temperatures. The sharp drop in friction is observed when the tip is pulled away from the surface and then the contact is restored. (b) Pull-off curves obtained in high friction regimes $(A, C)$ and low-friction regimes $(B, D)$, revealing significant reduction in adhesion after renewing the contact. 
temperatures. The transfer of metal atoms from the clean metal surface to a neck around the tip apex has been predicted in simulations [5] and observed in transmission electron microscopy [7], indentation experiments [6], and break junction experiments [18]. Our results show that such gold necks between tip and surface grow when sliding on $\mathrm{Au}(111)$ at temperatures below $170 \mathrm{~K}$, such that friction increases and the contact is plastically deformed under sliding. A small contact with low friction can be reestablished by pulling the tip out of contact. The formation of such a nanometer-sized contact with optimized strength in pull-off experiments has been predicted in simulations [19]. The rearrangement of the tip apex out of contact may be supported by the fact that in our setup the tip is cooled only while in contact with the surface [20].

The growth of the contact at low temperatures is associated with a particular distortion of the lateral force. Figure 3(a) shows a lateral force loop with high friction recorded after $150 \mathrm{~s}$ of continuous scanning at $30 \mathrm{~K}$. Large
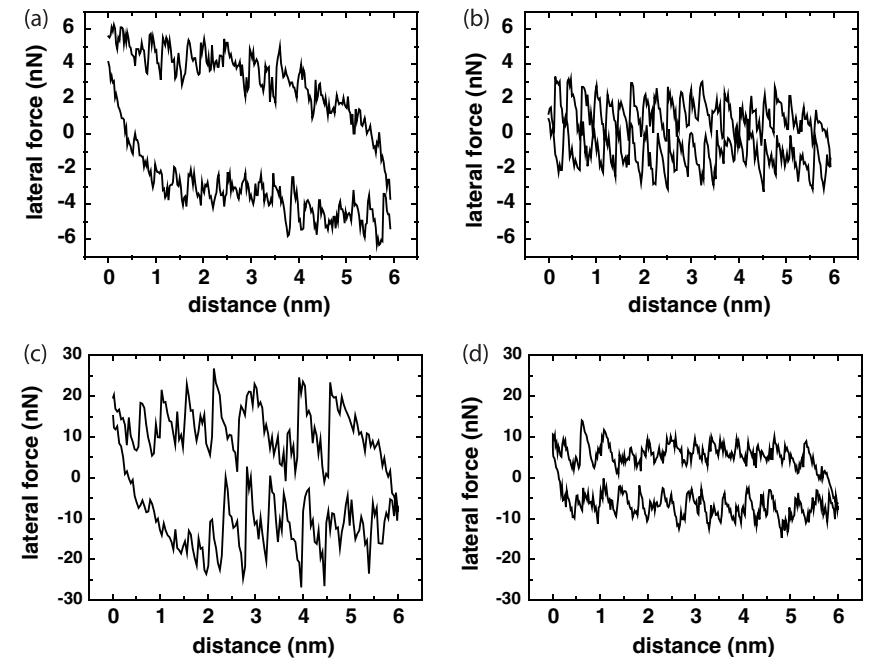

(e)

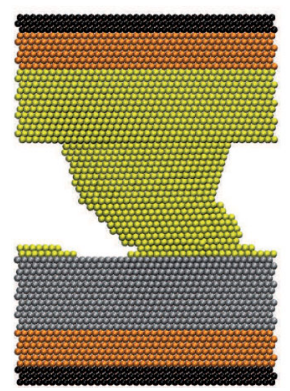

(f)

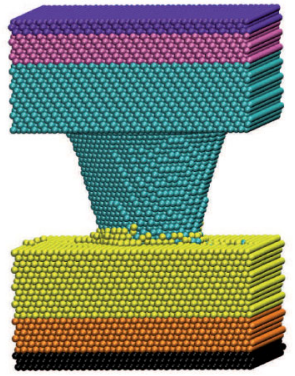

FIG. 3 (color online). Lateral force loop recorded before (a) and after (b) the pull-off experiment in Fig. 2 are compared to lateral force loops from the simulation of a $\mathrm{Au}(111)$ (c) and a $\mathrm{Ni}(111)$ (d) tip in commensurate contact with a $\mathrm{Au}(111)$ surface. Both simulated lateral curves are the third forward and backward loop of the respective simulation. Snapshots from the atomistic simulation of the Au and Ni cone tips are displayed in (e) and (f), respectively. The shading of the balls indicates fixed atoms of the support, atoms in the thermostat region, and atoms which can move freely. hysteresis, rather irregular stick slip, and a bending of the lateral force curve are observed in this high-friction regime. The low-friction lateral force loop in Fig. 3(b) is recorded just after the pull-off experiment labeled $B$ in Fig. 2(a). Stick slip with the periodicity of the $\mathrm{Au}(111)$ surface lattice and a straight force curve are obtained in this low-friction regime. Lateral force loops recorded at temperatures higher than $170 \mathrm{~K}$ are always similar to Fig. 3(b).

In order to relate these lateral force curves to microscopic mechanisms, we performed atomistic simulations of gold and nickel tips sliding on the $\mathrm{Au}(111)$ surface. Note, that a more realistic simulation of a silicon oxide tip sliding in contact with a gold substrate would require an extremely sophisticated empirical interatomic potential or even a full quantum mechanical treatment. Both are not feasible at the moment and therefore we restricted our simulations to gold-nickel model systems. Our simulation setup consists of a $10 \mathrm{~nm} \times 10 \mathrm{~nm} \times 5 \mathrm{~nm} \mathrm{Au(111)} \mathrm{slab} \mathrm{and} \mathrm{a} \mathrm{truncated}$ conical tip with a $3 \mathrm{~nm}$ diameter circular end in a simulation box with horizontal periodic boundary conditions. Interatomic forces have been derived from a second moment tight-binding potential, parametrized for gold-nickel multilayer systems [21]. Newton's equations of motion describing the atomic dynamics have been integrated using a velocity Verlet algorithm with a time step of $5 \mathrm{fs}$. The substrate and the tip holder have been terminated by freezing their 3 last atomic layers and coupling the adjacent 6 layers to a Langevin thermostat for the transverse degrees of freedom. The frozen atoms of the substrate have been fixed, whereas the frozen atoms of the tip holder have been coupled to a barostat [22] and used to record the normal and friction force. Each tip-substrate system has first been relaxed at zero contact pressure. The tip has then been scanned by applying a velocity of $2 \mathrm{~m} / \mathrm{s}$ to the frozen atoms of the tip holder while keeping the barostat pressure to zero. The scanning direction has been periodically reverted after a scanning distance of $6 \mathrm{~nm}$. Each simulation has been run for at least 5 half cycles, corresponding to a simulation time of $15 \mathrm{~ns}$.

First, we consider the sliding of the $\mathrm{Au}(111)$ tip in commensurate contact with the $\mathrm{Au}(111)$ surface [Fig. 3(e)] at $T=0 \mathrm{~K}$. We obtained lateral force curves that show all characteristic features of the experimental force curve recorded at low temperatures [Fig. 3(a)], namely, the large hysteresis, the irregular stick slip and the bent appearance. Analysis of the simulation trajectories reveals the mechanisms behind the bending of the force curve. Upon change of scanning direction, the Au tip is first elastically deformed, giving rise to the linear part of the force curve. The tip is then plastically deformed by atomic glide events parallel to the substrate surface. In this simulation, the $\mathrm{Au}$ tip is an ideal fcc single crystal during the forward scan, but exhibits a deformation twin during the backward scan, as demonstrated in the simulation snapshot in Fig. 3(e). The bending of the force curve reveals the formation and 
healing of the deformation twin. The same tip rotated by an angle of $18.3^{\circ}$ around the vertical axis with respect to the substrate did also show stick-slip behavior, but with lower friction and without a bend in the force curve (not shown). Thus, the experimentally observed friction loops in the high friction regime encountered at low temperatures exhibit all features of a larger gold junction sliding in commensurate contact over the surface.

Regular low-friction stick-slip curves are also observed, when the commensurate gold tip is replaced by a Ni tip of approximately the same size and with the same crystallographic orientation [see Fig. 3(f) for a snapshot from a $T=300 \mathrm{~K}$ simulation]. In this case the absence of a bent friction loop [Fig. 3(d)] can be explained by the mismatch of the Au and Ni lattice constant and the increased stiffness of the Ni tip. Interestingly, the simulated lateral force curve in Fig. 3(d) compares well to the experimental result in Fig. 3(b). Similar stick-slip behavior reflecting the atomic structure of (111) metal surfaces has been observed in simulations before for very small tips of $\mathrm{Cu}$ sliding on $\mathrm{Cu}(111)$ [23] and of Pt sliding on $\mathrm{Au}(111)$ [17]. We conclude that the regular stick-slip observed in our experiments indicates that the silicon tip apex carries either a small stiff Au nanotip in commensurate contact or a small $\mathrm{Au}$ transfer layer making contact in a near-commensurate fashion with the $\mathrm{Au}(111)$ surface.

The significant differences in the frictional properties above and below $170 \mathrm{~K}$ are then caused by the growth of a commensurate Au neck between tip and $\mathrm{Au}(111)$ surface at lower temperatures. At higher temperatures, we observe constant friction together with regular stick-slip. We believe that diffusion of surface atoms plays a crucial role in establishing the stable frictional behavior. At $170 \mathrm{~K}$ and above, surface diffusion of $\mathrm{Au}$ atoms is fast enough to replenish any damage on the $\mathrm{Au}(111)$ surface and to effectively lubricate the sliding nanotip or nearcommensurate contact. This effect is confirmed by our observation that nanometer-scale scratches in the $\mathrm{Au}(111)$ surface heal within a few scan frames under continuous scanning in contact [24]. The results indicate that the stable frictional behavior at elevated temperatures relies on a dynamic equilibrium at the sliding contact, where eventual damages of the $\mathrm{Au}(111)$ surface are quickly healed by diffusion of $\mathrm{Au}$ atoms. Lowering the temperature below $170 \mathrm{~K}$ leads to a drop in diffusion rates of gold surface atoms which prevents the self-healing of eventual defects formed under the sliding tip, resulting in the growth of a commensurate Au neck between tip and surface. The drop of surface diffusion at $170 \mathrm{~K}$ could be caused by the freeze-out of detachment-attachment mechanisms of gold intralayer diffusion. It was observed that the mechanisms leading to nanoscale ripple formation when sputtering $\mathrm{Au}(111)$ stop operating at similar temperatures [25]. Simulation of the effects of surface atom diffusion on the time scale of our experiment is beyond the limits of computation power. Our attempts to include the effects of diffusion through simulation at very high temperatures and corresponding rescaling of the simulation time [26] failed due to the expected mechanical softening of the tips which rendered high-temperature simulations incomparable with low-temperature experiments.

To summarize, stable stick-slip friction with atomic periodicity is found on $\mathrm{Au}(111)$ in the temperature range between 300 and $170 \mathrm{~K}$. The weak dependence on velocity and temperature is in agreement with basic thermal activation models of atomic-scale jumps of the contact. At temperatures below $170 \mathrm{~K}$, an increase in friction with time is observed. Comparison with molecular dynamics simulations suggests that the growth of a gold contact and its plastic deformation during sliding lead to increasing friction observed at low temperatures. The regular stick-slip behavior with low friction observed at room temperature is actually the result of a dynamic equilibrium requiring the diffusion of surface atoms in the contact region.

The authors acknowledge Dr. Lars Jansen and Gernot Langewisch for useful discussions and help in the experiments. N. N. G. acknowledges support from the Alexandervon-Humboldt Foundation. The project is financially supported by the Deutsche Forschungsgemeinschaft through the ESF Eurocores Programme FANAS.

[1] M.J. Madou, Fundamentals of Microfabrication: The Science of Miniturization (CRC Press, Boca Raton, Florida, 2002), 2nd ed..

[2] F. P. Bowden and D. Tabor, The Friction and Lubrication of Solids (Clarendon, Oxford, 1986).

[3] I. Szlufarska, M. Chandross, and R. W. Carpick, J. Phys. D 41, 123001 (2008).

[4] E. Meyer, R. M. Overney, K. Dransfeld, and T. Gyalog, Nanoscience: Friction and Rheology on the Nanometer Scale (World Scientific, Singapore, 1998).

[5] U. Landman, W. D. Luedtke, N. A. Burnham, and R. J. Colton, Science 248, 454 (1990).

[6] R. C. Thomas, J.E. Houston, T. A. Michalske, and R. M. Crooks, Science 259, 1883 (1993).

[7] A. P. Merkle and L. D. Marks, Wear 265, 1864 (2008).

[8] R. Bennewitz et al., Phys. Rev. B 60, R11301 (1999).

[9] M. Enachescu, R. W. Carpick, D. F. Ogletree, and M. Salmeron, J. Appl. Phys. 95, 7694 (2004).

[10] N. N. Gosvami, T. Filleter, P. Egberts, and R. Bennewitz, Tribol. Lett. 39, 19 (2010).

[11] R. C. Jaklevic and L. Elie, Phys. Rev. Lett. 60, 120 (1988).

[12] A. Schirmeisen, L. Jansen, H. Hölscher, and H. Fuchs, Appl. Phys. Lett. 88, 123108 (2006).

[13] L. Jansen, A. Schirmeisen, J. L. Hedrick, M. A. Lantz, A. Knoll, R. Cannara, and B. Gotsmann, Phys. Rev. Lett. 102, 236101 (2009).

[14] X. Zhao, S. R. Phillpot, W. G. Sawyer, S. B. Sinnott, and S. S. Perry, Phys. Rev. Lett. 102, 186102 (2009).

[15] I. Barel, M. Urbakh, L. Jansen, and A. Schirmeisen, Phys. Rev. Lett. 104, 066104 (2010). 
[16] E. Gnecco, R. Bennewitz, T. Gyalog, C. Loppacher, M. Bammerlin, E. Meyer, and H. J. Güntherodt, Phys. Rev. Lett. 84, 1172 (2000).

[17] Q. Li, Y. Dong, D. Perez, A. Martini, and R. W. Carpick, Phys. Rev. Lett. 106, 126101 (2011).

[18] G. Rubio-Bollinger, S. R. Bahn, N. Agraït, K. W. Jacobsen, and S. Vieira, Phys. Rev. Lett. 87, 026101 (2001).

[19] H. Kim and A. Strachan, Phys. Rev. B 83, 024108 (2011).

[20] X. Zhao, S. R. Phillpot, W. G. Sawyer, S. B. Sinnott, and S. S. Perry, Phys. Rev. Lett. 105, 229602 (2010).

[21] T. Deutsch, P. Bayle, F. Lancon, and J. Thibault, J. Phys. Condens. Matter 7, 6407 (1995).
[22] L. Pastewka, S. Moser, and M. Moseler, Tribol. Lett. 39, 49 (2010).

[23] M. R. Sørensen, K. W. Jacobsen, and P. Stoltze, Phys. Rev. B 53, 2101 (1996).

[24] See Supplemental Material at http://link.aps.org/ supplemental/10.1103/PhysRevLett.107.144303 for results demonstrating self-healing of wear damage on $\mathrm{Au}$ (111) induced by surface diffusion.

[25] G. Bracco and D. Cavanna, Phys. Rev. B 76, 033411 (2007).

[26] M. Moseler, F. Cervantes-Sodi, S. Hofmann, G. Csanyi, and A. C. Ferrari, ACS Nano 4, 7587 (2010). 